PROCEEDINGS OF THE

AMERICAN MATHEMATICAL SOCIETY

Volume 125, Number 11, November 1997, Pages 3185-3189

S 0002-9939(97)04112-9

\title{
A NOTE ON HENSEL'S LEMMA IN SEVERAL VARIABLES
}

\author{
BENJI FISHER
}

(Communicated by Wolmer V. Vasconcelos)

\begin{abstract}
The standard hypotheses for Hensel's Lemma in several variables are slightly stronger than necessary, in the case that the Jacobian determinant is not a unit. This paper shows how to weaken the hypotheses for Hensel's Lemma and some related theorems.
\end{abstract}

\section{INTRODUCTION}

The most familiar version of Hensel's Lemma states that if $f$ is a polynomial with coefficients in $\mathbb{Z}_{p}, a \in \mathbb{Z}_{p}$ is an approximate root of $f($ i.e., $f(a) \equiv 0(\bmod p))$, and $f^{\prime}(a)$ is a unit $\left(\right.$ i.e., $\left.f^{\prime}(a) \not \equiv 0(\bmod p)\right)$ then there is a unique root $a^{\prime}$ near $a$ : $f\left(a^{\prime}\right)=0$ and $a^{\prime} \equiv a(\bmod p)$. The first application of this theorem is to the polynomial $f(X)=X^{p}-X$ : by Fermat's Little Theorem, every $p$-adic integer $a$ is an approximate root of $f$; clearly, $f^{\prime}(a) \equiv-1(\bmod p)$ for all $a \in \mathbb{Z}_{p}$. Thus there are $p$ roots of this polynomial in $\mathbb{Z}_{p}$; they are known as the Teichmüller representatives of the residue classes $(\bmod p)$.

One way to generalize Hensel's Lemma is to relax the requirement that $f^{\prime}(a)$ be a unit. Let $h=v_{p}\left(f^{\prime}(a)\right)$. Letting $r=v_{p}(f(a))$, one can show that if $r>2 h$ then there is a unique root $a^{\prime}$ such that $a^{\prime} \equiv a\left(\bmod p^{r-h}\right)$. The first example of this version of Hensel's Lemma is extracting square roots 2 -adically: if $a^{2} \equiv c\left(\bmod 2^{r}\right)$, with $c$ odd and $r>2$, then there is a square root of $c$ congruent to $a\left(\bmod 2^{r-1}\right)$. In particular, an odd number has a square root in $\mathbb{Z}_{2}$ if and only if it is congruent to $1(\bmod 8)$.

A further generalization is to consider $n$ polynomial equations in $n$ variables. The analogous statement, with the derivative replaced by the Jacobian determinant, is true. Let bold-face letters denote $n$-tuples: $\mathbf{a}=\left(a_{1}, \ldots, a_{n}\right)$ and so on. Let $\mathbf{f}$ be an $n$-tuple of polynomials in $n$ variables and $J_{\mathbf{f}}=\partial\left(f_{1}, \ldots, f_{n}\right) / \partial\left(X_{1}, \ldots, X_{n}\right)$ the Jacobian determinant. Let $\mathbf{a} \in \mathbb{Z}_{p}{ }^{n}$ and $h=v_{p}\left(J_{\mathbf{f}}(\mathbf{a})\right)$. If $r=\min _{i}\left\{v_{p}\left(f_{i}(\mathbf{a})\right)\right\}>$ $2 h$ then there is a unique $\mathbf{a}^{\prime} \in \mathbb{Z}_{p}{ }^{n}$ such that $f_{i}\left(\mathbf{a}^{\prime}\right)=0$ and $a_{i}^{\prime} \equiv a_{i}\left(\bmod p^{r-h}\right)$ for all $i$.

The point of this note is that the hypothesis $r>2 h$ above is stronger than necessary. The first improvement is to note how $h=v_{p}\left(J_{\mathbf{f}}(\mathbf{a})\right)$ is used in the proof: by Cramer's Rule, the Jacobian matrix times its adjoint (or adjoint-transpose) has the form $p^{h} \times($ unit $) \times I_{n}$; in other words, the Jacobian matrix $M_{\mathbf{f}}(\mathbf{a})$ divides $p^{h} I_{n}$. Any value of $h$ for which this is true will work just as well as $v_{p}\left(J_{\mathbf{f}}(\mathbf{a})\right)$. For example,

Received by the editors May 20, 1996.

1991 Mathematics Subject Classification. Primary 13J15; Secondary 13J05, 13B40.

Key words and phrases. Hensel's lemma, power series, Henselian rings.

(C) 1997 American Mathematical Society 
if $M_{\mathbf{f}}(\mathbf{a})$ is diagonal, say $M_{\mathbf{f}}(\mathbf{a})=\operatorname{diag}(p, \ldots, p, 1)$ then one can take $h=1$ instead of $h=v_{p}\left(J_{\mathbf{f}}(\mathbf{a})\right)=n-1$, a big improvement. Sometimes it is convenient to allow larger values of $h$.

The second improvement is to note that the hypothesis that $\mathbf{f}(\mathbf{a})$ be divisible by $p^{2 h+1}$ can be replaced by the hypothesis that $\mathbf{f}(\mathbf{a})$ be divisible by $p^{h+1} M_{\mathbf{f}}(\mathbf{a})$, in the sense of matrix multiplication: that is, $\mathbf{f}(\mathbf{a})=p^{h} M_{\mathbf{f}}(\mathbf{a}) \cdot \mathbf{b}$ for some $\mathbf{b} \in\left(p \mathbb{Z}_{p}\right)^{n}$. For example, again suppose that $M_{\mathbf{f}}(\mathbf{a})$ is diagonal, say $M_{\mathbf{f}}(\mathbf{a})=\operatorname{diag}\left(p^{h}, 1, \ldots, 1\right)$ (so that the first improvement had no effect). Instead of requiring that $f_{i}(\mathbf{a}) \equiv 0$ $\left(\bmod p^{2 h+1}\right)$ for all $i$, one may require this for $i=1$ and $f_{i}(\mathbf{a}) \equiv 0\left(\bmod p^{h+1}\right)$ for all $i>1$.

For a number-theoretic application of this improved form of Hensel's Lemma, see my paper with R. Dabrowski, [Da-F, Theorem 1.8]. I would be interested to see a more geometric application. In the situation described above, the proof is given in [Da-F, Lemma 1.20]. In the rest of this paper, I will verify that the same improvements can be made in more general settings.

I would like to thank Romuald Dabrowski for his insistence, while we were preparing $[\mathrm{Da}-\mathrm{F}]$, that we give definitive statements of our results. It was this insistence that forced me to formulate this improved version of Hensel's Lemma.

\section{Definitions and notations}

Following Bourbaki [B2, §III.4.5], I will say that a commutative ring $A$ and an ideal $\mathfrak{m} \subseteq A$ satisfy Hensel's conditions if $A$ is complete and separated with respect to a linear topology (i.e., a topology defined by ideals) and $\mathfrak{m}$ is closed, consisting of topologically nilpotent elements. (In the Introduction, $A=\mathbb{Z}_{p}$ and $\mathfrak{m}=p \mathbb{Z}_{p}$.) To avoid confusion with the $n^{\text {th }}$ power of $\mathfrak{m}$, the $n$-fold Cartesian product of $\mathfrak{m}$ with itself will be denoted $\mathfrak{m}^{(n)}$. (In [B2], this is denoted $\mathfrak{m}^{\times n}$.)

Let $A\{\mathbf{X}\}$ denote the ring of restricted (formal) power series in $n$ variables, where $\mathbf{X}=\left(X_{1}, \ldots, X_{n}\right)$ [B2, $\S$ III.4.2]. If $\mathbf{f}=\left(f_{1}, \ldots, f_{m}\right)$ is an $m$-tuple in $A\{\mathbf{X}\}$, let $M_{\mathbf{f}}$ denote the Jacobian matrix; if $m=n$, let $J_{\mathbf{f}}=\operatorname{det} M_{\mathbf{f}}$ be the Jacobian determinant. Let $M_{\mathbf{f}}^{(r)}$ denote the matrix consisting of the first $r$ columns of $M_{\mathbf{f}}$; let $M_{\mathbf{f}}^{(-r)}$ denote the matrix consisting of the last $r$ columns of $M_{\mathbf{f}}$. (In [B2], there is no notation for the first $r$ columns; the last $r$ columns are denoted $M_{\mathbf{f}}^{(r)}$.) In particular, $M_{\mathbf{f}}=\left[M_{\mathbf{f}}^{(r)} M_{\mathbf{f}}^{(r-n)}\right]$. Similarly, if $\mathbf{a} \in A^{m}$, let $\mathbf{a}^{(r)}$ and $\mathbf{a}^{(-r)}$ denote the first and last $r$ entries of $\mathbf{a}$. Let $\mathbf{1}_{n}$ denote the $n$-tuple $\mathbf{1}_{n}=\mathbf{X}=\left(X_{1}, \ldots, X_{n}\right)$, so that $M_{\mathbf{1}_{n}}=I_{n}$ (the identity matrix). I will always think of $\mathbf{f}$ and $\mathbf{a}$ as column vectors.

In particular, if $A$ is a discrete ring then it is automatically complete and separated; $\mathfrak{m}$ is automatically closed; and a restricted power series is simply a polynomial. In any case, polynomials are special cases of restricted power series.

\section{Hensel's Lemma in Several VARIABles}

Let $A$ be a ring and $\mathfrak{m} \subseteq A$ an ideal. I will assume that $A$ and $\mathfrak{m}$ satisfy the simplest version of Hensel's Lemma in several variables and derive a more general version that incorporates the points discussed in the Introduction. The argument closely follows one in Greenberg [Gr]. I will then show that if $A$ is a Henselian local ring and $\mathfrak{m}$ is its maximal ideal then this theorem applies. (This is surely well-known, but I do not know a reference.) 
Assume that $A$ is complete and separated and that $\mathfrak{m}$ is closed, with respect to a linear topology. Let us say that $(A, \mathfrak{m})$ satisfies "condition $(\mathrm{H})$ " if the following version of Hensel's Lemma holds:

Condition (H). Let $\mathbf{f}=\left(f_{1}, \ldots, f_{n}\right)$ be an n-tuple of restricted power series $f_{i} \in$ $A\{\mathbf{X}\}$ and let $J=J_{\mathbf{f}}$ be the Jacobian determinant. Let $\mathbf{a} \in A^{n}$; assume that $\mathbf{f}(\mathbf{a}) \in \mathfrak{m}^{(n)}$ and that $J(\mathbf{a}) \in A^{\times}$. Then there is a unique $\mathbf{a}^{\prime} \in A^{n}$ such that $\mathbf{f}\left(\mathbf{a}^{\prime}\right)=\mathbf{0}$ and $\mathbf{a}^{\prime} \equiv \mathbf{a}\left(\bmod \mathfrak{m}^{(n)}\right)$.

Remark. If we further assume that every element of $\mathfrak{m}$ is topologically nilpotent then $(A, \mathfrak{m})$ satisfy Hensel's conditions, and so condition $(\mathrm{H})$ is satisfied by [B2, $\S I I I .4 .5$, Corollaire 3] ( $c f . \S 3$, below).

Theorem 1. Assume that $(A, \mathfrak{m})$ satisfies condition $(\mathrm{H})$. Let $\mathbf{f}=\left(f_{1}, \ldots, f_{n}\right)$ be an n-tuple with $f_{i} \in A\{\mathbf{X}\}$. Let $\mathbf{a} \in A^{n}$ and let $e \in A$ be such that $M_{\mathbf{f}}(\mathbf{a}) \cdot M^{\prime}=e I_{n}$ for some matrix $M^{\prime}$ (with entries in $A$ ). Assume that $\mathbf{f}(\mathbf{a}) \in e M_{\mathbf{f}}(\mathbf{a}) \mathfrak{m}^{(n)}$ : i.e., $f(\mathbf{a})=e M_{\mathbf{f}}(\mathbf{a}) \cdot \mathbf{b}$ for some $\mathbf{b} \in \mathfrak{m}^{(n)}$. Then there is some $\mathbf{a}^{\prime} \in A^{n}$ such that $\mathbf{f}\left(\mathbf{a}^{\prime}\right)=\mathbf{0}$ and $\mathbf{a}^{\prime} \equiv \mathbf{a}(\bmod e \mathfrak{m})$. If $e$ is not a zero-divisor then $\mathbf{a}^{\prime}$ is unique.

Proof. For a single formal power series $f \in A[[\mathbf{X}]]$, Taylor's theorem [B1, $\S \mathrm{IV} .5 .8$, Proposition 9] gives

$$
f(\mathbf{X}+\mathbf{Y})=f(\mathbf{X})+M_{f}(\mathbf{X}) \cdot \mathbf{Y}+\sum_{1 \leq i \leq j \leq n} G_{i j}(\mathbf{X}, \mathbf{Y}) Y_{i} Y_{j}
$$

for some $G_{i j} \in A[[\mathbf{X}, \mathbf{Y}]]$. Arguing as in [B2, §III.4.5], if $f$ is restricted then so are the entries $\partial f / \partial X_{i}$ of $M_{f}$ and the $G_{i j}$. Therefore, taking $f=f_{i}$, one can replace $\mathbf{X}$ with $\mathbf{a}$ and $\mathbf{Y}$ with $e \mathbf{X}$, obtaining

$$
\mathbf{f}(\mathbf{a}+e \mathbf{X})=\mathbf{f}(\mathbf{a})+M_{\mathbf{f}}(\mathbf{a}) \cdot e \mathbf{X}+e^{2} \mathbf{R}(\mathbf{X})=e M_{\mathbf{f}}(\mathbf{a}) \cdot\left[\mathbf{b}+\mathbf{X}+M^{\prime} \mathbf{R}(\mathbf{X})\right],
$$

where the remainder terms satisfy $R_{i} \in(\mathbf{X})^{2} A\{\mathbf{X}\}$. Let $\mathbf{h}(\mathbf{X})=\mathbf{b}+\mathbf{X}+M^{\prime} R(\mathbf{X})$ and apply condition $(\mathrm{H})$ to $\mathbf{h}$ : since $M_{\mathbf{h}}(\mathbf{0})=I_{n}$ and $\mathbf{h}(\mathbf{0})=\mathbf{b} \in \mathfrak{m}^{(n)}$, there is a unique $\mathbf{x} \in \mathfrak{m}^{(n)}$ such that $\mathbf{h}(\mathbf{x})=\mathbf{0}$. Let $\mathbf{a}^{\prime}=\mathbf{a}+e \mathbf{x}$, so that $\mathbf{a}^{\prime} \equiv \mathbf{a}\left(\bmod e \mathfrak{m}^{(n)}\right)$ and $\mathbf{f}\left(\mathbf{a}^{\prime}\right)=\mathbf{0}$.

Now assume that $e$ is not a zero-divisor. Since $M_{\mathbf{f}}(\mathbf{a}) \cdot M^{\prime}=e I_{n}, J_{\mathbf{f}}(\mathbf{a})=$ $\operatorname{det} M_{\mathbf{f}}(\mathbf{a})$ is also not a zero-divisor. If $\mathbf{a}^{\prime}=\mathbf{a}+e \mathbf{x}$ is a root of $\mathbf{f}$, with $\mathbf{x} \in \mathfrak{m}^{(n)}$, then multiplying both sides of Equation (1) (with $\mathbf{X}$ replaced by $\mathbf{x}$ ) by the adjoint of $M_{\mathbf{f}}(\mathbf{a})$ gives $\mathbf{0}=e J(\mathbf{a}) \mathbf{h}(\mathbf{x})$, which implies $\mathbf{h}(\mathbf{x})=\mathbf{0}$. This proves uniqueness.

Proposition 2. Let $A$ be a (discrete) local ring with maximal ideal $\mathfrak{m}$. Then $A$ is Henselian if and only if $(A, \mathfrak{m})$ satisfy condition $(\mathrm{H})$.

Proof. Assume that $A$ is a Henselian local ring. The first step is to reduce to the case $n=1$, using the structure theory of étale $A$-algebras. Following the notation of Raynaud [R], let $C=A[\mathbf{X}], I=(\mathbf{f}) \subseteq C$, and $B=(C / I)_{J}=A[\mathbf{X}, T] /(\mathbf{f}, T J-1)$. If $R$ is any $A$-algebra then $\operatorname{Hom}_{A}(B, R)$ is the set of $\mathbf{a} \in R^{n}$ such that $\mathbf{f}(\mathbf{a})=\mathbf{0}$ and $J(\mathbf{a}) \in R^{\times}$. Thus we must show that the canonical map $\operatorname{Hom}_{A}(B, A) \longrightarrow$ $\operatorname{Hom}_{A}(B, k)$ is an isomorphism, where $k=A / \mathfrak{m}$.

According to the Jacobian criterion $[\mathrm{R}, \mathrm{V}$, Théorème 5$]$ and the fact that being étale is a local condition [R, II, Proposition 6], $B$ is an étale $A$-algebra. The question is local on Spec $B$ : choosing a homomorphism $\phi: B \longrightarrow k$ determines a prime ideal $\mathfrak{q}=\operatorname{ker} \phi$, and lifting $\phi$ to a map $B \longrightarrow A$ is the same as lifting it to a map 
$B_{g} \longrightarrow A$, for any $g \in B-\mathfrak{q}$. By the local structure theorem [R, $\mathrm{V}$, Théorème 1$]$, one can choose $g$ so that $B_{g}$ is isomorphic to a standard étale $A$-algebra:

$$
B_{g} \cong B^{\prime}=(A[X] /(f))_{h}
$$

for a single monic polynomial $f \in A[X]$ (in one variable), where $f^{\prime}$ is invertible in $B^{\prime}$. This completes the reduction step.

The surjectivity of $\operatorname{Hom}_{A}\left(B^{\prime}, A\right) \longrightarrow \operatorname{Hom}_{A}\left(B^{\prime}, k\right)$ is just a translation of [R, VII, Proposition 3]. Injectivity follows from a standard argument: if $f(a+x)=f(a)=0$ with $x \in \mathfrak{m}$ then Taylor's theorem gives $x\left[f^{\prime}(a)+G(a, x) x\right]=0$. Since $f^{\prime}(a)$ is a unit and $x \in \mathfrak{m}$, the quantity in brackets is a unit, which implies that $x=0$.

Conversely, if $(A, \mathfrak{m})$ satisfy condition $(\mathrm{H})$ then, taking $n=1$, the converse direction of [R, VII, Proposition 3] shows that $A$ is Henselian.

\section{The Inverse and Implicit Function Theorems}

Theorems 3 and 4 below are improvements of Théorème 2 and Corollaire 3 of [B2, $\S$ III.4.5], to which one may refer for details of the proofs. Theorem 3 is an algebraic version of the Inverse Function Theorem; Theorem 4 is an algebraic version of the Implicit Function Theorem. Taking $r=0$ in Theorem 4 gives Hensel's Lemma in several variables. (Instead of re-proving this theorem, one can apply Theorem 1 to produce an exact root and then use the version in [B2].)

Theorem 3. Let $A$ and $\mathfrak{m}$ satisfy Hensel's conditions. Let $\mathbf{f}$ be an $n$-tuple of restricted power series $f_{i} \in A\{\mathbf{X}\}$ and let $\mathbf{a} \in A^{n}$. Let $e \in A$ and let $M^{\prime}$ be an $n \times n$ matrix (with entries in $A$ ) such that $M_{\mathbf{f}}(\mathbf{a}) \cdot M^{\prime}=e I_{n}$. There is an n-tuple $\mathbf{g}$, with $g_{i} \in(\mathbf{X}) A\{\mathbf{X}\}$, such that

(i) $M_{\mathbf{g}}(0)=I_{n}$.

(ii) For all $\mathbf{x} \in A^{n}$,

$$
\mathbf{f}(\mathbf{a}+e \mathbf{x})=\mathbf{f}(\mathbf{a})+M_{\mathbf{f}}(\mathbf{a}) \cdot e \mathbf{g}(\mathbf{x}) .
$$

(iii) Let $\mathbf{h}$ be the $n$-tuple of formal power series (not necessarily restricted) $h_{i} \in$ $A[[\mathbf{X}]]$ such that $\mathbf{g} \circ \mathbf{h}=\mathbf{1}_{n}$. For all $\mathbf{y} \in \mathfrak{m}^{(n)}$,

$$
\mathbf{f}(\mathbf{a}+e \mathbf{h}(\mathbf{y}))=\mathbf{f}(\mathbf{a})+M_{\mathbf{f}}(\mathbf{a}) \cdot e \mathbf{y} .
$$

Proof. Taking $\mathbf{g}=\mathbf{1}_{n}+M^{\prime} \cdot \mathbf{R}$, (i) and (ii) follow from Equation (1); and (iii) follows by replacing $\mathbf{x}$ with $\mathbf{h}(\mathbf{y})$.

Theorem 4. Let $A$ and $\mathfrak{m}$ satisfy Hensel's conditions. Let $\mathbf{f}=\left(f_{r+1}, \ldots, f_{n}\right)$ be an $(n-r)$-tuple of restricted power series $f_{i} \in A\{\mathbf{X}\}$ and let $\mathbf{a} \in A^{n}$. Let $e \in A$ and let $M^{\prime}$ be an $(n-r) \times(n-r)$ matrix (with entries in $A$ ) such that $M_{\mathbf{f}}^{(r-n)}(\mathbf{a}) \cdot M^{\prime}=e I_{n-r}$. Assume that $\mathbf{f}(\mathbf{a})=e M_{\mathbf{f}}^{(r-n)}(\mathbf{a}) \cdot \mathbf{b}$ for some $\mathbf{b} \in \mathfrak{m}^{(n-r)}$. Then there are $n-r$ power series $\phi_{i} \in\left(\mathbf{X}^{(r)}\right) A\left[\left[\mathbf{X}^{(r)}\right]\right](r<i \leq n)$ such that, for all $\mathbf{t} \in \mathfrak{m}^{(r)}$,

$$
\mathbf{f}\left(\mathbf{a}^{(r)}+e^{2} \mathbf{t}, \mathbf{a}^{(r-n)}+e \phi(\mathbf{t})\right)=0
$$

Proof. Apply Theorem 3 to $\mathbf{u}=\left(\mathbf{X}^{(r)}-\mathbf{a}^{(r)}, \mathbf{f}\right)$. Note that $M_{\mathbf{u}}=\left[\begin{array}{cc}I_{r} & 0 \\ M_{\mathbf{f}}^{(r)} & M_{\mathbf{f}}^{(r-n)}\end{array}\right]$, so that $M_{\mathbf{u}}(\mathbf{a})\left[\begin{array}{cc}e I_{r} & 0 \\ -M^{\prime} M_{\mathbf{f}}^{(r)}(\mathbf{a}) & M^{\prime}\end{array}\right]=e I_{n}$. It follows that, in Equation (1), $G_{i j}=0$ and $R_{i}=0$ for $1 \leq i \leq r$; and, in the proof of Theorem $3, g_{i}=h_{i}=X_{i}$. 
According to Theorem 3(iii),

$$
\mathbf{u}(\mathbf{a}+e \mathbf{h}(\mathbf{y}))=\mathbf{u}(\mathbf{a})+M_{\mathbf{u}}(\mathbf{a}) \cdot e \mathbf{y}=M_{\mathbf{u}}(\mathbf{a}) \cdot e\left[\begin{array}{c}
\mathbf{y}^{(r)} \\
\mathbf{b}+\mathbf{y}^{(n-r)}
\end{array}\right] .
$$

Thus $\mathbf{f}(\mathbf{a}+e \mathbf{h}(\mathbf{y}))=\mathbf{0}$ if and only if $e\left(M_{\mathbf{f}}^{(r)}(\mathbf{a}) \cdot \mathbf{y}^{(r)}+M_{\mathbf{f}}^{(r-n)}(\mathbf{a}) \cdot\left(\mathbf{b}+\mathbf{y}^{(r-n)}\right)\right)=\mathbf{0}$. To guarantee this, it suffices to set $\mathbf{y}^{(r)}=e \mathbf{t}$ and $\mathbf{y}^{(r-n)}=-\mathbf{b}-M^{\prime} M_{\mathbf{f}}^{(r)}(\mathbf{a}) \cdot \mathbf{t}$, with $\mathbf{t} \in \mathfrak{m}^{(r)}$. Therefore set $\phi\left(\mathbf{X}^{(r)}\right)=\mathbf{h}^{(r-n)}\left(e \mathbf{X}^{(r)},-\mathbf{b}-M^{\prime} M_{\mathbf{f}}^{(r)}(\mathbf{a}) \cdot \mathbf{X}^{(r)}\right)$; the theorem follows.

\section{REFERENCES}

[B1] N. Bourbaki, Algèbre, Hermann, Paris, 1959.

[B2] - Algèbre Commutative, Hermann, Paris, 1962.

$\left[\right.$ Da-F] R. Dabrowski and B. Fisher, A stationary-phase formula for exponential sums over $\mathbb{Z} / p^{m} \mathbb{Z}$ and applications to GL(3)-Kloosterman sums, Acta. Arith. (to appear).

[Gr] M. J. Greenberg, Rational points in Henselian discrete valuation rings, Pub. Math. IHES 31 (1966), 59-64. MR 34:7515

[R] M. Raynaud, Anneaux Locaux Henseliens, Lecture Notes in Math. 169, Springer-Verlag, Berlin-Heidelberg-New York, 1970. MR 43:3252

Department of Mathematics, Columbia University, New York, New York 10027

Current address: The Bronx High School of Science, 75 West $205^{\text {th }}$ Street, Bronx, New York 10468

E-mail address: benji@math.columbia.edu 